\title{
Improving promptness of antibiotic treatment in meningococcal disease
}

\author{
F A I Riordan
}

\begin{abstract}
Objectives-Delays in parenteral antibiotic treatment may contribute to the high mortality in meningococcal disease. This study aimed to record "door to needle" time in children with meningococcal disease before and after the introduction of a specific teaching programme about the disease.

Methods-"Door to needle" time in 33 children with meningococcal disease, admitted June 1995-December 1996, were studied. Regular teaching sessions encouraging prompt treatment were started in January 1997. "Door to needle" time was then studied for 42 children admitted January 1997-December 1998.

Results-More of the second cohort attended accident and emergency (A\&E) directly ( 9 of $33 v 24$ of $42 ; p=0.01$ ) rather than being referred by a GP. Similar proportions received pre-admission antibiotics from a GP (8 of $24 v 5$ of 18). Mortality was similar in the two groups ( 2 of $33 v 5$ of 42). "Door to needle" time was significantly shorter in the second cohort in those with a typical rash (median $60 \mathrm{~min}$ utes $v 18$ minutes; $p=0.0004$ ). Only 1 of 23 (4\%) children in the second cohort with a typical, petechial rash waited more than 60 minutes for antibiotics, compared with 6 of 24 in the first cohort $(p=0.06)$.

Conclusions-Significant improvements in "door to needle" time in meningococcal disease can be achieved when awareness is heightened by regular teaching. Those with a typical, petechial rash can be treated within 60 minutes of arrival. Strategies to improve immediate treatment of meningococcal disease should include education of $A \& E$ staff, especially as an increasing proportion of cases present directly to $A \& E$. (Emerg Med f 2001;18:162-163)
\end{abstract}

Keywords: meningococcal infection; child; antimicrobial therapy

Department of Child Health, Birmingham Heartlands and Solihull NHS Trust (Teaching), Bordesley Green East,

Birmingham B9 5SS, UK

Correspondence to: Dr Riordan

(evansk@)

heartsol.wmids.nhs.uk)

Accepted for publication September 2000

Early recognition and treatment of meningococcal disease is recommended to decrease mortality. ${ }^{12}$ General practitioners (GPs) and accident and emergency (A\&E) staff are advised to give parenteral penicillin to cases of suspected meningococcal disease "as soon as the diagnosis is suspected". ${ }^{3}$ However preadmission antibiotics are only given to $30 \%$ of those admitted by GPs. ${ }^{4}$ In urban areas many patients with meningococcal disease present directly to A\&E departments. ${ }^{5}$ The majority of patients will thus have not received parenteral antibiotics before arrival at hospital.

When awareness of meningococcal disease is high, all those with a typical rash receive parenteral antibiotics within one hour of arrival. ${ }^{5}$ However, in routine practice antibiotics are delayed for more than one hour in 37\% of those with a typical rash. ${ }^{6}$ Delays in antibiotic treatments also occur in $15 \%$ of those who die from meningococcal disease and may contribute to the poor outcome. ${ }^{1}$

The aim of this study was to record "door to needle" time in children with meningococcal disease before and after the introduction of a specific teaching programme about the disease.

\section{Methods}

This prospective study included all children seen with meningococcal disease at Birmingham Heartlands and Solihull Trust between June 1995 and December 1998. The Trust has two A\&E departments (at Heartlands and Solihull hospitals), but all paediatric admissions are at Heartlands. During the study period all paediatric admissions were initially assessed in A\&E at Heartlands. Meningococcal disease was defined using the Meningococcal Disease Surveillance Group criteria. ${ }^{7}$ Disease severity was assessed using the Glasgow Meningococcal Septicaemia Prognostic Score. "Door to needle" time was defined as the time from the recorded time of arrival at hospital until the parenteral administration of appropriate antibiotics. "Typical rash" was defined as a petechial or purpuric rash on arrival at hospital and excluded children with no rash or maculopapular rashes. Time of administration of antibiotics was determined from GP referral letters, A\&E cards, drug charts, case notes or directly from the staff involved.

From January 1997 a regular teaching session about meningococcal disease was given to nurses and doctors in $\mathrm{A} \& \mathrm{E}$ and paediatrics. This was a lecture using slides, which focused on recognition of the rash and the importance of prompt antibiotic treatment. "Door to needle" times, for those with typical rash and those with atypical or no rash, were compared before and after this date.

\section{Results}

Seventy five children with meningococcal disease were seen during the study period (69 confirmed microbiologically); seven died. The children's ages, disease severity and mortality did not differ significantly between the two time periods (table 1 ). Significantly more children presented directly to $\mathrm{A} \& \mathrm{E}$, rather than via 
Table 1 Comparison of children seen with meningococcal disease before and after introduction of a specific teaching programme about the disease. Statistical comparisons by Mann-Whitney U test and Fisher's exact test

\begin{tabular}{llll}
\hline & $\begin{array}{l}\text { fune 1995-Dec 1996 } \\
(n=33)\end{array}$ & $\begin{array}{l}\text { fan 1997-Dec 1998 } \\
(n=42)\end{array}$ & $p$ Value \\
\hline Median age in years (range) & $1.8(0.3-15.5)$ & $3.5(0.2-15)$ & 0.9 \\
GP referral (pre-admission antibiotics) & $24(8)$ & $18(5)$ & 0.01 \\
Self referral (A\&E) & 9 & 24 & 0.96 \\
Median GMSPS (range) & $5(2-15)$ & $5(2-15)$ & 0.46 \\
Deaths & 2 & 5 & 0.21 \\
Door to needle time in minutes ${ }^{\star}$ median (range) & $90(15-1320)$ & $48(5-3000)$ & 0.0004 \\
Door to needle time in minutes ${ }^{\star}$ if typical rash on arrival & $60(15-240)$ & $18(5-66)$ & 0.73 \\
Door to needle time in minutes if atypical or no rash on arrival & $270(60-1320)$ & $150(30-3000)$ & \\
\hline
\end{tabular}

$\star 13$ children receiving pre-admission antibiotics excluded.

their GP in the second time period (table 1). For 45 children the first dose of parenteral antibiotics was given in the A\&E department.

In the first time period six (25\%) of the 24 with a typical rash, did not receive antibiotics within one hour. This decreased to one $(4 \%)$ of 23, after regular teaching sessions. "Door to needle" time was significantly shorter in the second cohort in those with a typical rash (median 60 minutes $v 18$ minutes; $\mathrm{p}=0.0004$ ) (table 1). "Door to needle" time did not differ between the two cohorts in those presenting with atypical or no rash (table 1).

\section{Discussion}

This study shows that increasing awareness of meningococcal disease at our Trust has led to significantly prompter antibiotic treatment for those with a typical rash. The study also shows an increasing proportion of children with meningococcal disease presenting directly to $\mathrm{A} \& \mathrm{E}$, rather than via their GP. "Door to needle" time only decreased in those with a typical rash. This suggests there was improved recognition of the rash, not of the sick child.

The main strength of this study was that it was prospective, ensuring good case ascertainment and accurate documentation of timing of antibiotics. In retrospective studies it may be difficult to determine when antibiotics were given. ${ }^{6} \mathrm{~A}$ further strength is that teaching about meningococcal disease was done by one person, ensuring a consistent message was given to staff. Awareness of meningococcal disease may also have been increased by a clinical effectiveness initiative studying "door to needle" time for all adults and children with meningococcal disease in Birmingham from July 1997 to June 1998. However, of those cases of meningococcal disease reported to the initiative only six of 18 with a rash received antibiotics within 60 minutes (A Wood, personal communication).

Awareness of meningococcal disease may also have been increased during the second period, by the rise in reported cases and heightened media coverage leading to increased awareness by parents. This may explain the increased direct presentation to $\mathrm{A} \& \mathrm{E}$. Other confounding variables are a change in the $A \& E$ triage system, the introduction of "Calman" specialist registrars and an increase in $\mathrm{A} \& \mathrm{E}$ consultants, during the study period.

This study did not record the number of children initially treated for meningococcal disease later found not to have the disease.
Increasing awareness of meningococcal disease may have increased this number and further studies in this group are needed. Earlier antibiotic treatment did not lead to a decrease in mortality in this study, but the number of deaths is too small for an effect to be evident. The increasing proportion of children with meningococcal disease presenting directly to $\mathrm{A} \& \mathrm{E}$ means less children have the opportunity to receive pre-admission penicillin. This makes prompt treatment in hospital even more important. Initiatives to increase awareness of meningococcal disease should be considered in all hospitals that see acutely unwell children.

Antibiotic treatment is only the first step in managing meningococcal disease. Assessment of disease severity and treatment of shock or raised intracranial pressure is also required. ${ }^{9}$ Delays in these areas of management may also affect outcome. ${ }^{2}$ Further studies looking at promptness of resuscitation and increasing awareness of meningococcal disease in other settings is needed. This study found "door to needle" time was unchanged in children with atypical or no rash. This suggests that further education about recognising sick children is needed.

In conclusion, strategies to improve immediate treatment of meningococcal disease should include regular education of both $\mathrm{A} \& \mathrm{E}$ and paediatric nursing and medical staff.

I would like to thank Wendy King for her enthusiasm, encouragement and for arranging many of the teaching sessions, Caroline Park and the other A\&E consultants for their support and Alistair Thomson for helpful comments.

\section{Contributor}

Andrew Riordan initiated the research, collected, analysed and interpreted the data and wrote the paper. Andrew Riordan acts as guarantor.

1 Slack J. Deaths from meningococcal infection in England and Wales in 1978. F R Coll Physicians London 1982;16:404 .

2 Nadel S, Britto J, Booy R, et al. Avoidable deficiencies in the delivery of health care to children with meningococcal disdelivery of health care to children with men

3 Donaldson L. Meningococcal infection. PL/CMO/99/1. London: DoH, 1999.

4 Cartwright K, Reilly S, White D, et al. Early treatment with parenteral penicillin in meningococcal disease. BMF 1992; 305:143-7.

5 Riordan FAI, Thomson APJ, Sills JA, et al. Prospective study of "door-to-needle time" in meningococcal disease. 7 Accid Emerg Med 1998;15:249-51.

6 Wood AL, O'Brien SJ. How long is too long? Determining the early management of meningococcal disease in Birmingham. Public Health 1996;110:237-9.

7 Meningococcal Disease Surveillance Group. Meningococcal disease: secondary attack rate and chemoprophylaxis in the United States, 1974. fAMA 1976;235:261-5.

8 Thomson APJ, Sills JA, Hart CA. Validation of the Glasgow Meningococcal Septicaemia Prognostic Score: a 10-year Meningococcal Septicaemia Prognostic Score: a 10-y
retrospective survey. Crit Care Med 1991;19:26-30.

9 Pollard AJ, Britto J, Nadel S, et al. Emergency management of meningococcal disease. Arch Dis Child 1999;80:290-6. 